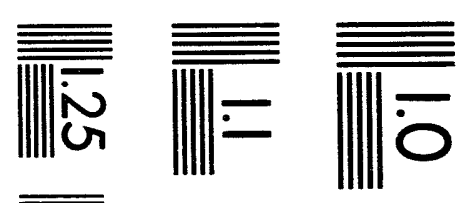

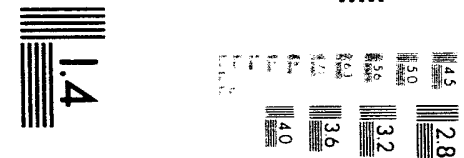

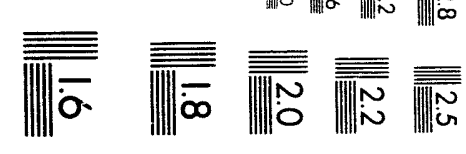



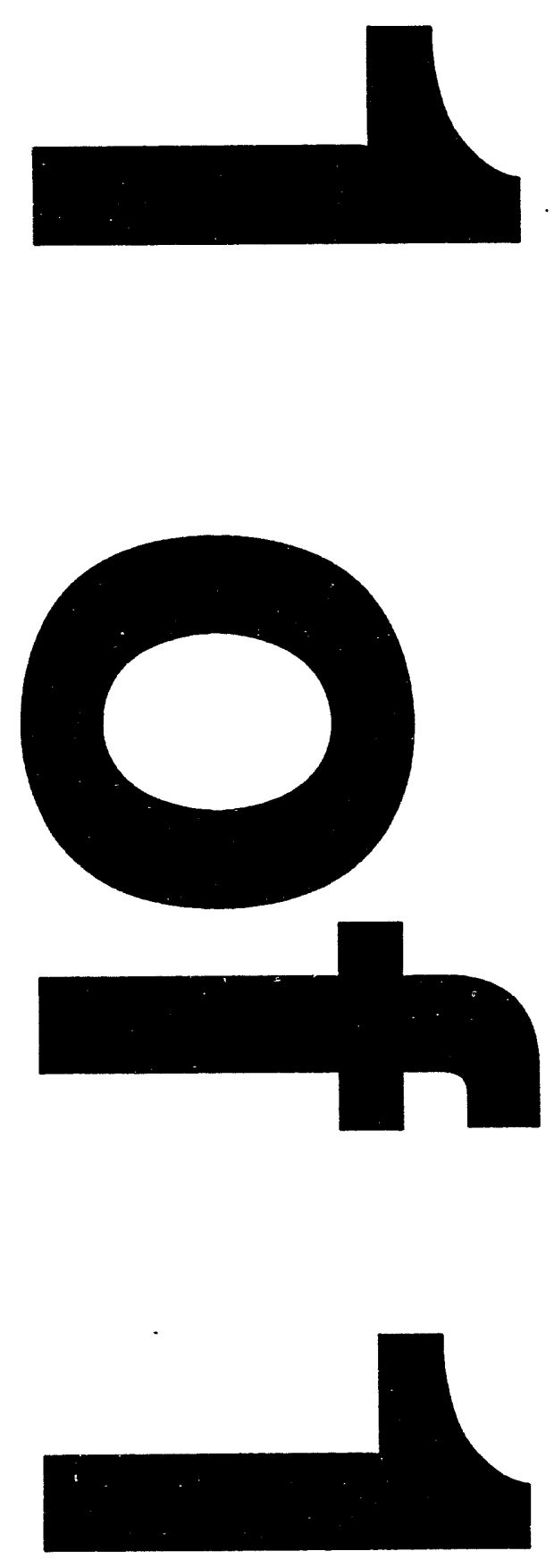


\title{
Eddy Current Control in the AGS Rapid Cycling Booster Accelerator Magnets*
}

\author{
G. T. Danby, J. W. Jackson and C. Spataro \\ Alternating Gradient Synchrotron Department, Brookhaven National Laboratory \\ Upton, New York 11973
}

\begin{abstract}
The Booster requires highly variable magnet cycles. When $B$ is large, eddy current induced sextupole, etc., in the dipole vacuum chamber (VC) is large, with a much smaller contribution from magnet ends. Simple passive coils excited automatically by transformer action cancel the $B$ induced sextupole. ${ }^{1}$ A seif correction coil is not required for the quadrupoles, since $B$ induced aberrations are very small ( $<1.0 \times 10^{-4}$ at full aperture). Iron magnetization does not produce dipole or quadrupole magnet multipole aberrations, so these magnets have been effectively made independent of unwanted multipoles for all cycles. However, variations in the transfer functions and thus the Booster tune have not been automatically eliminated. Iron magnetization contributions are almost matched, but the $B$ induced field retardation in the dipoles VC is larger than in the quadrupoles. Results of measurements will be presented, plus a simple system to overcome the mismatch and make the tune independent of $B$. Properties of special lattice magnets and their corrections will also be described.
\end{abstract}

\section{INTRODUCTION}

The Booster required $10^{\circ}$ curved vacuum chambers capable of high temperature bakeout." The simplest approach is to use reliable and economical heavy walled self supporting chambers in the 36 dipole magnets and throughout the accelerator. This approach is normally not used in rapid cycling machines because of the large eddy current fields generated. This problem was solved by devising windings attached to the vacuum chamber which are powered by transformer action from auxiliary windings around the magnet poles and which cancel the sextupole and higher even power multipoles induced in the chambers. The correction automatically self corrects at any rise rate used in the Booster. The series correction consists of 3 turns on the top and 3 on the bottom of each chamber, fabricated from ceramic insulated copper wire in a stainless steel outer jacket. This system succeeds in correcting aberrations, leaving a purely dipole eddy current field. ${ }^{1,3}$

The same approach could be applied to the 48 quadrupoles. However, dynamic measurements showed that correction was not necessary. ${ }^{4}$ Even for an acceptance equal to the entire aperture at injection field, the eddy current higher multipoles are $\leq 1 \times 10^{-4}$ of the quadrupole

- Worked performed under the auspices of the U.S. Dept. of Energy. field for any cycle of the Booster, without correction. Only quadrupolar eddy current field is generated.

In summary, the vacuum chamber induced fields have been reduced to dipole and quadrupole field suppression. In addition, $\mathrm{Fe}$ magnetization does not produce aberrations in either Booster magnet type, nor do magnet eddy current fields (in ends). ${ }^{4}$ This correction technique will work for a wide range of accelerators unless the frequency is so high that the vacuum chamber behaves diamagnetically. For slow cycling machines it would permit the use of economical aluminum vacuum chambers.

\section{Dipole AND Quadrupole Tracking Variation AND CONTROL}

As was summarized above, considerable effort was expended successfully to "linearize" the dipoles and quadrupoles of the Booster lattice. Over the required aperture the dipole integrated field is that of a pure dipole with $5^{\circ}$ geometric wedge angles on both ends. The quadrupole presents a linear gradient as sensed by a long integrating search coil. The very small limits on multipole aberrations holds for the magnets plus their vacuum chambers both statically and dynamically, including magnetization effects.

Variations in the magnet transfer functions have not been automatically eliminated, so if equal currents are used in the dipoles and quadrupoles the tune will vary somewhat for different cycles with their widely varying $B$ requirements.

In practice the quadrupoles have auxiliary tuning capability which those responsible for Booster commissioning and operations use to exercise tune control. Thus a lack of magnet tracking only adds another component to desirable auxiliary tune control for maximizing beam current, etc. Nevertheless it is attractive to have the capability to make the basic tune independent of cycle for series operation of dipoles and quadrupoles in fast cycling machines. The machine then behaves like the "Paper" design and adjustments of tune can be concentrated on questions of a fundamental nature impacting on optimum performance.

Various factors contribute to significant variations from a linear transfer function. Magnetization and saturation of the steel laminations can be adjusted in the maynet design so that dipoles and quadrupoles roughly track. Laminations effectively limit eddy current effects in the poles in two dimensions. However, magnet end design attempts to minimize eddy currents at magnet ends. This is particular-

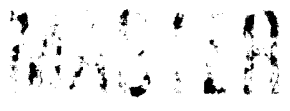

is

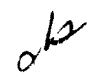

DISTRIBCUTIOR OI THIS DOCUMENT IS UN!IMITES

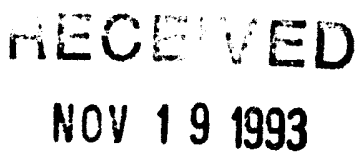


ly important for quadrupoles, which are short compared to dipoles.

In the Bcoster, dipole and quadrupole tracking differences are quite small, except for the effects of eddy currents in the vacuum chambers. As a result, on the rising cycle the gradient suppression $\Delta G / G$ is considerably smaller than the dipoles suppression $\Delta B / B$.

Figure 1 illustrates the method of measurement used. A long coil which extends beyond both ends of the dipole is shaped to measure the same dipole field integral traversed by a particle on the equilibrium orbit. A long gradient coil pair measures the integral field gradient straight through the quadrupole.

Between the two closely spaced magnets, the dipole coil is at the center line $r=0$ of the quadrupole, so it sees no quadrupole field. Similarly, the gradient pair is centered at $r=0$ in the quadrupole, so the dipole field sensed by each element of the pair are of opposite sign and also cancel. Small errors do contribute cross coupling of the dipole and quadrupole signals. However, by reversing the relative polarities of the dipole and quadrupole series excitation, these errors are measured and canceled by averaging, giving integral measurements of each magnet separately.

An air core solenoid containing an "Hcoil" search coil and a current transrlucer are operated in series with the dipole and quadrupole.

Table I shows the dipole field integral retardation from a linear transfer function as the rise rate is varied from the proton injection rise rate of $\dot{B}=12.9 \mathrm{kG} / \mathrm{sec}$ to the maximuin $\dot{B}=92 \mathrm{kC} / \mathrm{sec}$ which occurs at approximately $B_{0}=3.0 \mathrm{kG}$ in the dipole.

For this experiment, the quadruple was removed from the circuit and the long dipole integrating coil signal and the Hcoll signais were compr ed.

Ii Column 1, withcut the dipole vacuum chamber, the rise rate dependence is small and entirely associated with the dipole magnet end effects." Note again that the ruagnetization teld retarciation $\Delta B \sim 6$ gauss is almost pure dipole withcut significant multipole aberrations.

Column 2 shows the results with the vacuum chamber installed and multipole self correction operating to eliminate sextupole, etc., due to eddy currents. While the eddy current induced dipole is partially reduced,' vacuum Lamber eddy currents remain the largest source of field retardation $\Delta B$. In Table $I, \Delta B$ is negative because both magnetization and eddy currents cause the rising field to lag behind a linear transfer function.

In Table II the actual tracking of the dipole field integral ve"sus the quadrupole gradient integral is shown as a function of rise time. For this measurement the spacing of the gradient pair was adjusted to give the same total signal from this search coil as was obtained from the dipole search coil wher pulsed in series. These two signals were then added in series opposition so that for identical transfer functions the resultant signal would be zero.

Column 1 shows results without vacuum chambers in either magnet. When compared with Column 1 of Table I, this shows that the two magnets indeed almost track, so that the $\Delta G / G$ nearly matches $\Delta B / B$ for different rise rates.

Column 2 of Table II shows the results with vacuum chambers in both magnets and with self correction operating in the dipole. This is the situation that occurs in the Booster accelerator. Column 2 in Table II has smaller values than Column 2 in Table I, showing some comperisation. However, it is clear than $\triangle B / B$ in the dipole is considerably larger than $\Delta G / G$ in the quadrupole vacuum chamber. For example, consider a field retardation of the dipole with respect to the quadrupole of $\Delta B=-15$ gauss, occurring with $\dot{B}=90 \mathrm{kG} / \mathrm{sec}$ at $\mathrm{BO}=3 \mathrm{KG}$. This means that the cuadrupole ampere turns must be reduced by $15 / 3000=0.5 \%$ to match the two fields and restore the Booster to the nominal design tune value.

For the high rise rates typically used for the proton cycle of the Booster Figure 2 illustrates the dipole and quadruple tracking data. For this experiment, a triangular rise and fall of current was used, both with $\dot{B}= \pm 78 \mathrm{kG} / \mathrm{sec}$. The horizontal sweep is the current transducer signal using the excitation of the proton cycle, $640 \mathrm{amps}(1.6 \mathrm{kG})$ to $2200 \mathrm{amps}(5.5 \mathrm{kG})$. The upper part of the loop is the rising current, the only part of interest for the Booster accelerator.

The dotted rising curve is for illustration only, and was drawn onto the graph. It starts out at $\dot{B}=12 \mathrm{kG} / \mathrm{sec}$, which is the design value at injection, and then grows to qualitatively follow the increasing $\dot{B}$, reaching full value at approximately double the injection current, or $3 \mathrm{kG}$.

It would be possible to increase the eddy current suppression of the rising quadruple field so the resultant tracked the dipole. A thicker walled vacuum chamber or a more conductive chamber material could be used in the quadrupole. For example, with the dipole field increasing at $B=90 \mathrm{kG} / \mathrm{sec}$, introducing a $100 \mu \mathrm{sec}$ time delay into the quadruple field rise will shift the series signal by 9 gauss.

The 5 turns per pole primary quadrupole winding has an auxiliary circuit with a one turn per pole coil. Column 3 in Table II shows the results of applying a $71 \times 10^{-3} \mathrm{ohm}$ resistance in parallel with the auxiliary coil winding. Now $\Delta G / G$ and $\Delta B / B$ almost perfectly track for all rise rates.

Use of this technique for a fast pulsing machine can result in completely matched transfer functions so the tune of the machine will remain constant at all times, as in the "paper" design. Auxiliary tune variation need only carry. out desired manipulation in the tuning diamonds, as dictated by physics reasons. These results demonstrate this goal is practical using conventional thick walled vacuum chambers. Both the magnetization and the eddy current contributions track to high accuracy.

\section{SPECIAL AND CorRection MAGNets}

Beam injection into the AGS Booster occurs within a 
specially modified dipole magnet and its vacuum chamber. The injection line vacuum chamber is joined to the standard dipole vacuum chamber over an extended length near the longitudinal center of the dipole, welded at an angle of $12^{\circ}$ on the outside of the dipole chamber.

At the upstream end of the dipole the centerline of the injection line vacuum chamber is $40 \mathrm{~cm}$ outside of the centerline of the dipole aperture. This required modifying the dipole $\mathrm{H}$ magnet flux return, moving the return further from the poles to accommodate the pipe. The geometry of the return was modified to keep the reluctance and saturation unchanged as the path length through the iron return increased to accept the injection line.

The combined vacuum chamber in the junction region is considerably wider than the standard vacuum chamber and its center is displaced outward. Thus the eddy current induced field suppression is larger than normal and asymmetric. Figure 3 illustraies the field retardation measured with the long integrating curved coil traversing the entire dipole. Approximately $80 \mathrm{~cm}$ length of standard dipole vacuum chamber exists immediately upstream and downstream of the junction region. A self correction coil for sextupole was located on one end, and for quadrupole on the other end. The cross-section of the sextupole correction is identical to that in all standard full length dipoles. ${ }^{1,3}$ The quadrupole was achieved by locating ten equally spaced wires on the top and ten on the bottom of the vacuum chamber. The current direction is the same in all twenty wires, retuming through ten wires on both sides of the chamber. Figure 3 also shows the results of activating these self corrections, powered by windings around the pole ' by transformer action. A dipole self correction was also tested to make $\Delta B / B$ integrated through this special magnet equal to all the standard dipoles. However, the dipole difference is sufficiently small that this is not used in the Booster.

In summary, the injection dipole, like the standard dipoles, is operated in a manner where field moments other than dipole due to the vacuum chamber eddy currents are automatically canceled by transformer action.

Consider now magnetic field control elements. The Booster lattice ${ }^{2}$ has fully distributed sextupoles for chromaticity and sextupole control, one immediately adjacent to all 48 quadrupoles.

A multipurpose auxiliary magnet located in most half cells completes the magnetic elements. These small laminated iron core magnets surround the bellows of the vacuum system between each half cell so that they do not require any extra space. Windings on the core produce skew quadrupoles. Separate windings produce either vertical or horizontal dipole moments. Linear superposition holds, and high frequency excitation is practical.

No skew sextupole was originally built in. The Booster main magnets are iron dominated and very a : urate. The quadrupoles are four-fold, assembled from four separate pole pieces. The one piece laminations of the dipoles were stacked with four-fold rotation of lamination packs to average out stamping errors. Measurements of individual isolated magnets confirmed their accuracy, with skew moments $\leq 1 \times 10^{-4}$ of the main component.

In the actual Booster, various other sources of up-down asymmetry which can produce skew fields can occur due to interaction of magnets with the environment. In fact, the half cell bases under the magnets are made of iron, although an attempt was made to decouple them.

A study both experimental and computed was carried out to add skew sextupole windings to the auxiliary iron core magnets. This work is ongoing and successful.

It should be noted that prior to the recent record breaking high intensity proton run a relatively crude improvised insitu sextupole correction winding was added at only four locations. This immediately produced a large increase in proton intensity. ${ }^{5}$

Figure 4 illustrates a computed flux pattern for a winding design which will give essentially a pure skew sextupole, which will be applied, in the near future, to the auxiliary magnets. This correction has 12 turns in series as shown (actualiy 24 turns). Experiment shows that a slight modification gives the same pure sextupole integral result for the actual magnet. The laminated steel of the auxiliary magnet forms a square. Figure 4 lack of squareness is an artifact of reproduction. Also Figure 4 is rotated $45^{\circ}$ as indicated by the horizontal midplane (HMP). This correction will have a factor of 10 less dipole and higher multipole aberrations for a given skew sextupole strength.

Computations show that normal and skew octupole windings can easily be added to the auxiliary magnet.

These additions can provide a capability for control of all moments, normal and skew, up to and including octupole.

Experience of those responsible for operating the Booster illustrate that high intensities are very demanding. Even with great care tn all aspects of design and construction, to obtain the record intensities achiaved requires coping with very subtle effects. These additional corrections should prove valuable.

Table I

DIPOLE FIELD INTEGRAL VARIATION WITH RISE RATE

\begin{tabular}{|c|c|c|}
\hline Rise Rate & $\begin{array}{c}\text { Column 1 } \\
\text { No Vacuum Chamber* } \\
\Delta B \text { [Gauss] }\end{array}$ & $\begin{array}{c}\text { Column 2 } \\
\text { Vacuum Chamber** } \\
\Delta B \text { [Gauss] }\end{array}$ \\
\hline $\begin{array}{l}92 \mathrm{KG} / \mathrm{SEC} \\
\text { (Maximum) }\end{array}$ & -7.6 & -28.1 \\
\hline $47 \mathrm{KG} / \mathrm{SEC}$ & -0.6 & -10.3 \\
\hline $\begin{array}{l}12.9 \mathrm{KG} / \mathrm{SEC} \\
\text { (Proton Injection) }\end{array}$ & .6 .2 & -8.4 \\
\hline
\end{tabular}

- Without vacuum chamber, dipole has small varation of $A B$ with rise rate. The rise rate dependence is associated with magnet ends. The $\Delta \mathrm{B}$ is predominantly iron magnetization.

* The vacuum chamber has self-correction of sextupole in operation. the vacuum chamber eddy currents are now dominant. 
Table II

TRACKING OF DIPOLE FIELD INTEGRAL VS

QUADRUPOLE GRADIENT DNTEGRAL AS FUNCTION OF RISE RATE

\begin{tabular}{|l|c|c|c|}
\hline Rise Rate & $\begin{array}{c}\text { Column 1 } \\
\text { No Vacuum } \\
\text { Chamber }\end{array}$ & $\begin{array}{c}\text { Column 2 } \\
\text { Vacuum } \\
\text { Chamber }\end{array}$ & $\begin{array}{c}\text { Column 3 } \\
\text { Vacuum Chamber** } \\
+ \text { Quad Shunt }\end{array}$ \\
\hline $\begin{array}{l}92 \mathrm{KG} / \mathrm{SEC} \\
\text { (Macimom) }\end{array}$ & $-0.95 \mathrm{G}$ & $-16.80 \mathrm{G}$ & $0.03 \mathrm{G}$ \\
\hline $47 \mathrm{KG} / \mathrm{SEC}$ & $-0.27 \mathrm{G}$ & $-8.05 \mathrm{G}$ & $0.02 \mathrm{G}$ \\
\hline $\begin{array}{l}12.9 \mathrm{KG} / \mathrm{SEC} \\
\text { (njaction) }\end{array}$ & $0.24 \mathrm{G}$ & $-1.42 \mathrm{G}$ & $0.02 \mathrm{G}$ \\
\hline
\end{tabular}

Column 2 * Dipole vacuum chambers have self-correction coil for sextupole, etc., operating.

Column 3 * 71 Milliohm resistance applied across terminals of 1 turn per pole auxiliary quad coil. This column illustrates how tune could be made independent of $B$. Note that this is not present in the Booster, i.e. Column 2 applies.

References:

1. G.T. Danby and J.W. Jackson, "Vacuum Chamber Eddy Current Self-Correction for the AGS Booster Accelerator", Particle Accelerators, Vol. 27, pp 33-38, 1990.

2. Booster Design Manual, BNL, October, 1988.

3. G.T. Danby and J.W. Jackson, "Description of New Vacuum Chamber Correction Concept", 1989 Particle Accelerator Conf., Chicago, IL., March 20-23, BNL. -41856

4. G.T. Danby and J.W. Jackson, "Static and Dynamic Magnetic End Effects and Correction Magnets for the AGS Booster", 1989 Particle Accelerator Conf., Chicago, IL., March 20-23, BNL-41855.

5. C. Gardner and Coworkers, Private Communication 1993.

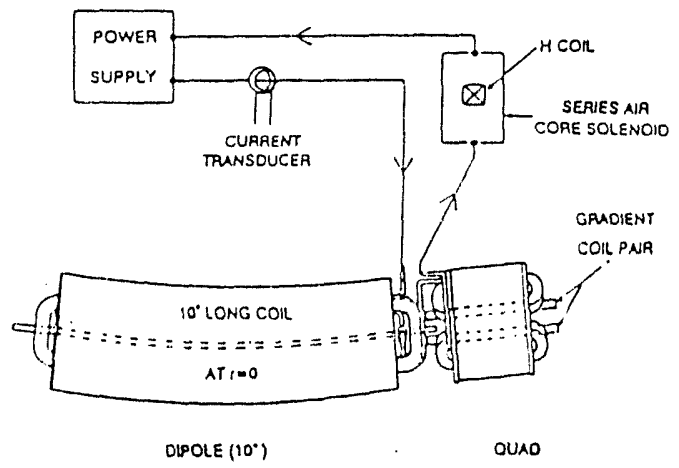

Fig. 1. Schematic representation of dipole and quadrupole measurement.

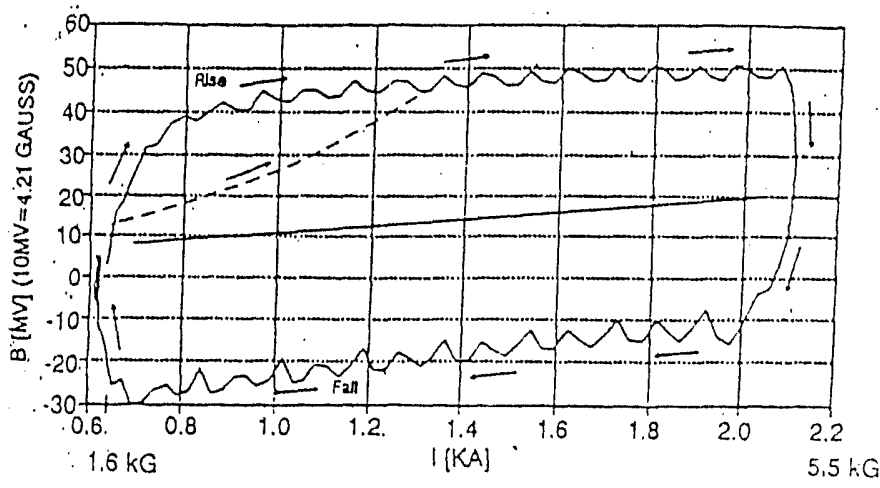

Fig. 2. Dipole and quadrupole tracking for the proton cycle

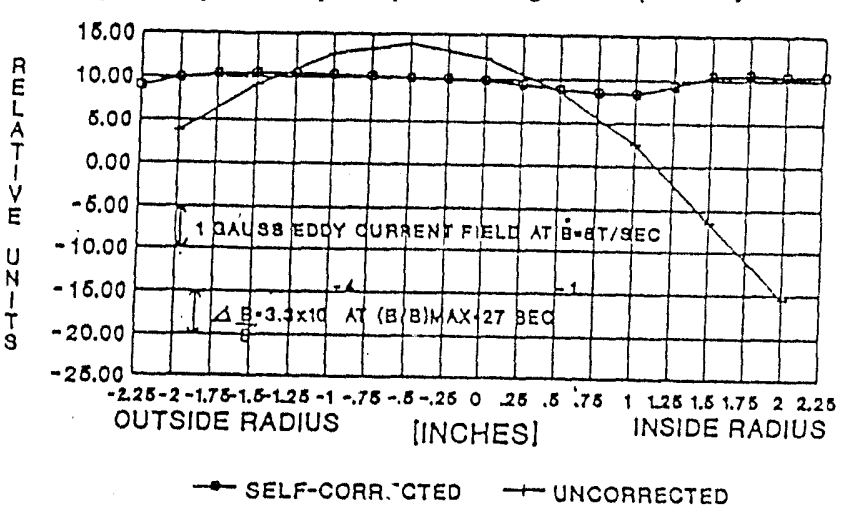

Fig. 3. Vacuum chamber eddy current field versus position.

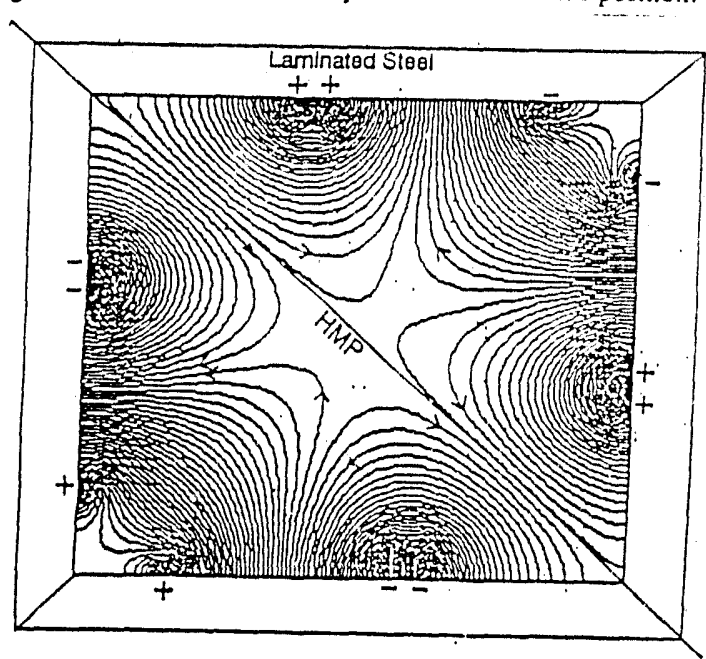

Fig. 4. Flux pattem for a skew sextupole coil winding.

\section{Acknowledgements}

The authors are indebted to J. Weisenbloom and E. Hoey for their great contribution to carrying out these experiments. 


\section{DISCLAIMER}

This report was prepared as an account of work sponsored by an agency of the United States Government. Neither the United States Government nor any agency thereof, nor any of their employees, makes any warranty, express or implied, or assumes any legal liability or responsibility for the accuracy, completeness, or usefulness of any information, apparatus, product, or process disclosed, or represents that its use would not infringe privately owned rights. Reference herein to any specific commercial product, process, or service by trade name, trademark, manufacturer, or otherwise does not necessarily constitute or imply its endorsement, recommendation, or favoring by the United States Government or any agency thereof. The views and opinions of authors expressed herein do not necessarily state or reflect those of the United States Government or any agency thereof. 

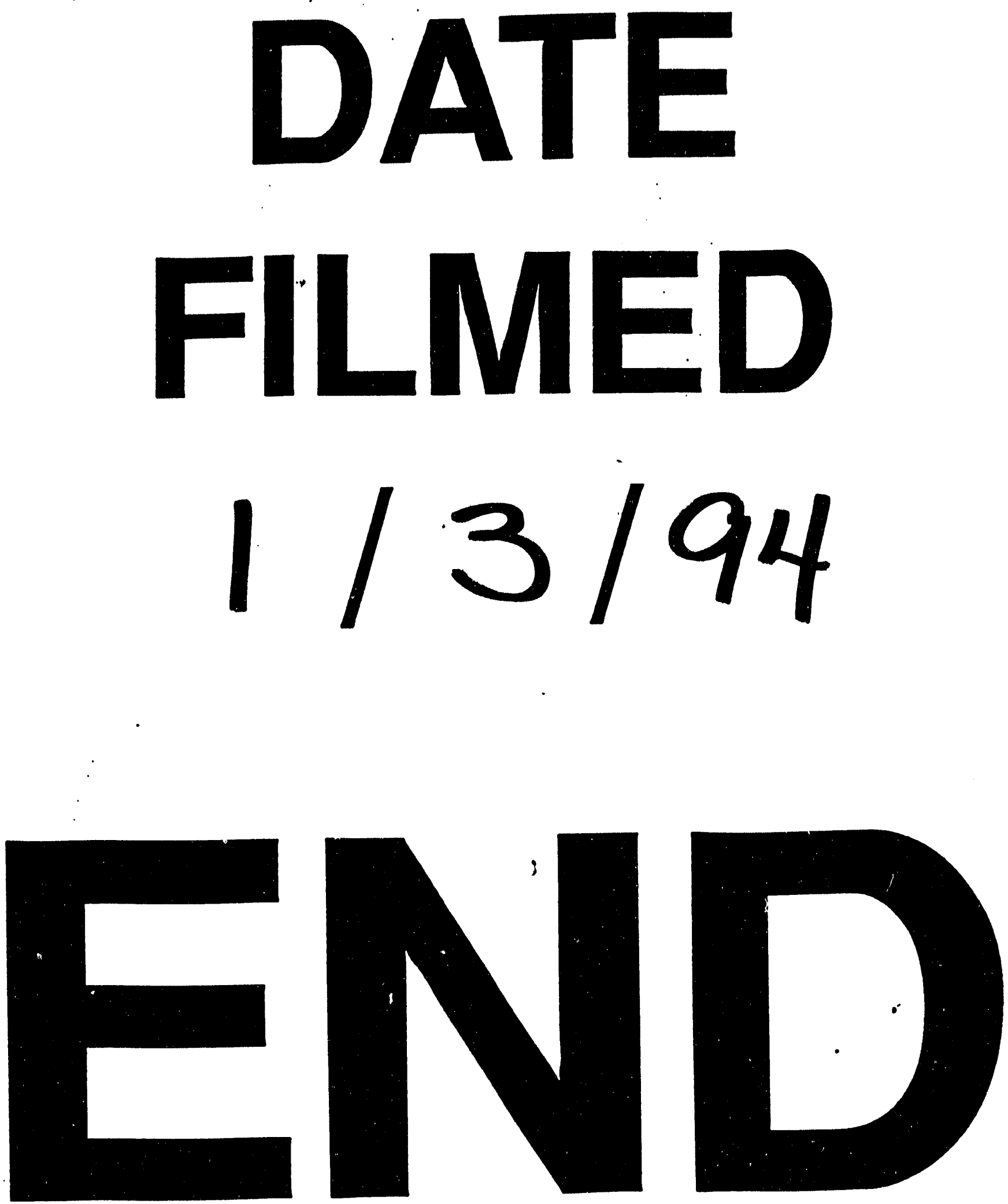


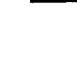

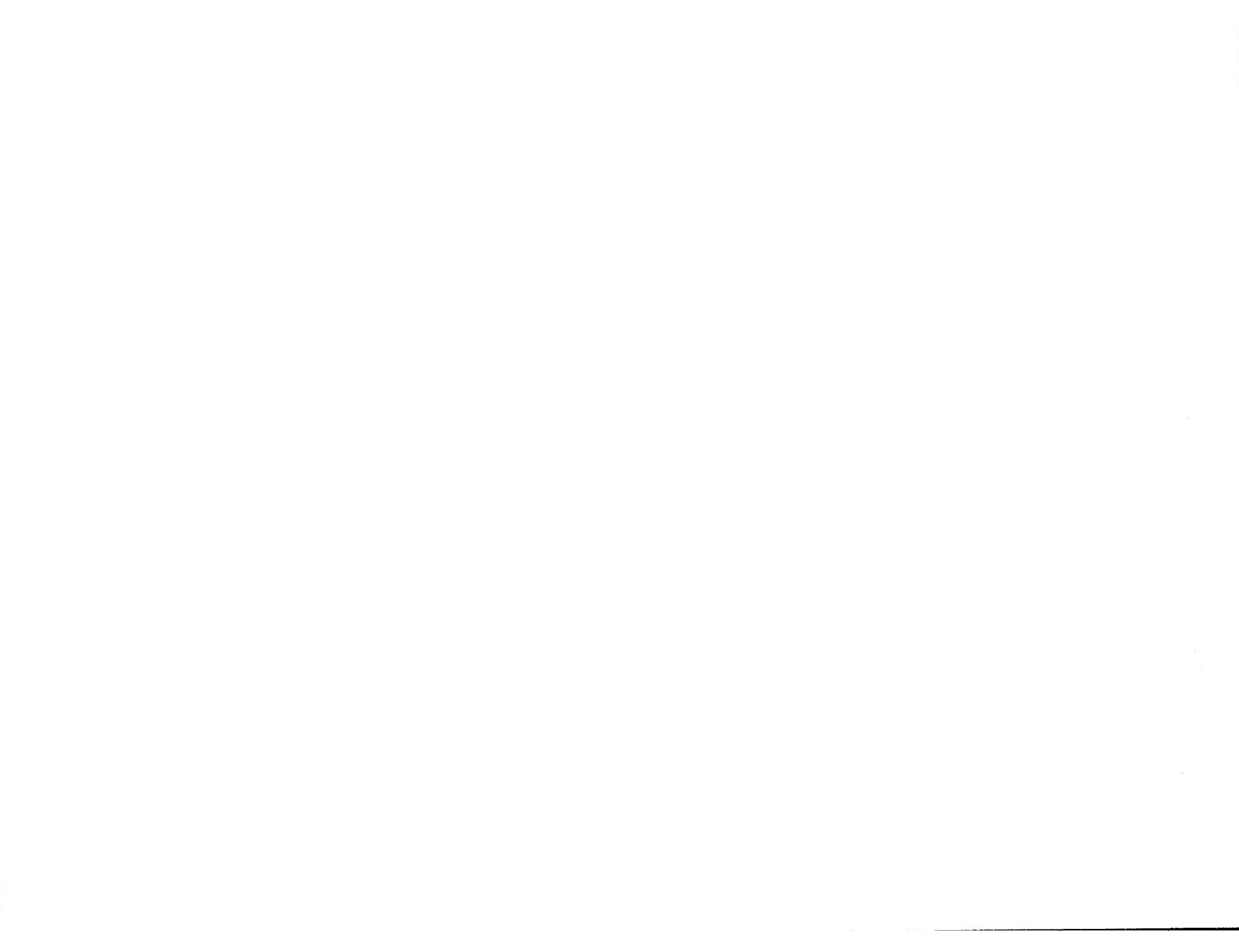

